\title{
Performance and Internal Flow of Contra-Rotating Small-Sized Cooling Fan
}

\author{
Toru Shigemitsu1 ${ }^{1}$, Keisuke Miyazaki², Katsuhiko Hirosawa², Hiroaki Fukuda² \\ ${ }^{1}$ Institute of Science and Technology, Tokushima University, Tokushima, Japan \\ ${ }^{2}$ Graduate School of Advanced Technology and Science, Tokushima University, Tokushima, Japan \\ Email: t-shige@tokushima-u.ac.jp
}

How to cite this paper: Shigemitsu, T. Miyazaki, K., Hirosawa, K. and Fukuda, H. (2018) Performance and Internal Flow of Contra-Rotating Small-Sized Cooling Fan. Open Journal of Fluid Dynamics, 8, 181-194.

https://doi.org/10.4236/ojfd.2018.82013

Received: May 2, 2018

Accepted: June 26, 2018

Published: June 29, 2018

Copyright () 2018 by authors and Scientific Research Publishing Inc. This work is licensed under the Creative Commons Attribution International License (CC BY 4.0).

http://creativecommons.org/licenses/by/4.0/

\begin{abstract}
High pressure and large flow rate small-sized cooling fans are used for servers in data centers and there is a strong demand to increase its performance because of increase of quantity of heat from servers. Contra-rotating rotors have been adopted for some of high pressure and large flow rate cooling fans to meet the demand. The performance curve of the contra-rotating small-sized cooling fan with $40 \mathrm{~mm}$ square casing was investigated by an experimental apparatus and its internal flow condition was clarified by the numerical analysis. The fan static pressure of the front rotor was extremely low and it increased significantly at the rear rotor. The uniform flow was achieved at the inlet of the rear rotor because of the special shape of the casing between the front and rear rotors. On the other hand, the tip leakage flow was large enough to influence on the main flow of the test cooling fan by the design specification of high pressure with compact rotor diameter.
\end{abstract}

\section{Keywords}

Cooling Fan, Small-Sized Axial Fan, Contra-Rotating Rotors, Performance, Internal Flow

\section{Introduction}

Data center has been built because of spread of cloud computing, establishment of ubiquitous networking society and increase of electric parts in machines. Then, power consumption in data centers, IT devices and machines has been increasing significantly [1]. Electrical power used for cooling of IT devices for data centers is as huge as that used for IT devices itself in data centers and the electric power consumption of it is growing rapidly. In the point of view of global warming and energy savings, there is a strong demand for reduction of power 
consumption in above facilities and equipments [2]. Axial fans are used as cooling fans of servers and desk top computers. Then, researches of axial fans on fan noise and effect of inlet geometry were conducted [3] [4]. High pressure and large flow rate small-sized cooling fans are used for servers in data centers and there is a strong demand to increase its pressure and flow rate because of increase of quantity of heat from servers. The increase of the pressure and flow rate by increase of a fan diameter is restricted because of limitation of space. Therefore, high rotational speed design is conducted, and rotational speed over $10,000 \mathrm{~min}^{-1}$ is employed for cooling fans of servers. Contra-rotating rotors have been adopted for some of high pressure and large flow rate cooling fans to meet its demand. On the other hand, low rotational speed design [5] and advantages on the performance of contra-rotating fans and pumps were verified by experimental results [6] [7] [8].

The company's research and development period for the contra-rotating small-sized cooling fan is short and its internal flow condition is not clarified well. Therefore, the performance of each front and rear rotor and internal flow condition were investigated by the numerical analysis with the simple numerical model without the spokes [9] [10]. In the case of the contra-rotating rotors, it is necessary to design the rear rotor considering the unsteady circumferential velocity distributions at the outlet of the front rotor [11]. Ravelet et al. investigated the effect of the blade loading of counter rotating axial fan on the performance and clarified the different circumferential velocity distributions at off design flow rate for each different blade loading types [12]. Further the fan noise becomes larger than the conventional rotor stator type fan because of the interaction between the front and rear rotors. Then, the passive noise reductions with the perforated blade were proposed for the contra-rotating fan [13]. It is important to clarify the influence of the wake from the front rotor to the rear rotor and the potential interaction between the front and rear rotors to increase the performance and to reduce the fan noise [14]. The blade row distance between front and rear rotors is a key parameter to consider the wake and potential interaction for the contra-rotating fan. The influence of the blade row distance between the front and rear rotors and pressure fluctuation on the casing wall were investigated for the counter rotating fan with fan diameter $D=375 \mathrm{~mm}$ [8] [15]. On the other hand, the conventional design method and the theory for the turbo machinery should be modified for small-sized axial fans because small-sized axial fans applied to electrical devices belong to extremely small size field in the turbo machinery [16]. Therefore, there is the strong demand to establish the design method for small-sized axial fans based on the internal flow between the front and rear rotors. Furthermore, the performance characteristics of each front and rear rotor and internal flow condition of the contra-rotating small-sized cooling fan having the spokes are not clarified well. Therefore, a design method of the contra-rotating small-sized cooling fan is not established.

In the present paper, the performance characteristics of each front and rear rotor of contra-rotating small-sized axial fan with $40 \mathrm{~mm}$ square casing are 
shown by the experimental and numerical results. Then, the internal flow conditions of the contra-rotating small-sized cooling fan having spokes are discussed based on the numerical analysis results.

\section{Experimental Apparatus and Method}

A picture of the exterior and primary dimensions of the high pressure and large flow rate contra-rotating small-sized cooling fan (R40W-A) are shown in Figure 1 and Table 1 respectively. The rotors of R40W-A are set in a $40 \mathrm{~mm}$ square casing, and the hub tip ratio of the front and rear rotors are $D_{h} / D_{t f}=25$ $\mathrm{mm} / 37.2 \mathrm{~mm}=0.67$ and $D_{h r} / D_{t r}=26 \mathrm{~mm} / 37.2 \mathrm{~mm}=0.70$ respectively. Operating flow rate is $Q_{o}=0.55 \mathrm{~m}^{3} / \mathrm{min}$ and a tip clearance is $c=0.6 \mathrm{~mm}$. Each rotational speed of the front and rear rotors of R40W-A is extremely high as $N_{f}=$ $15,000 \mathrm{~min}^{-1}$ and $N_{r}=14,000 \mathrm{~min}^{-1}$. Figure 2 shows a schematic diagram of an

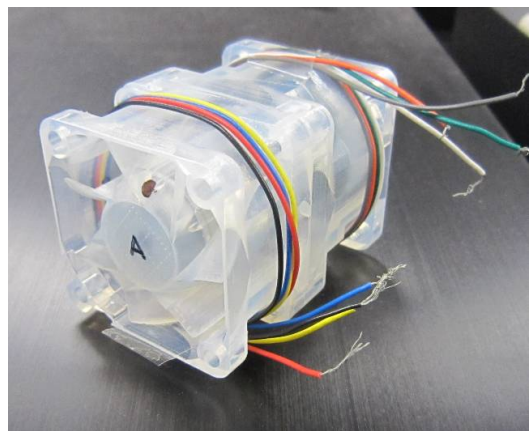

Figure 1. Picture of exterior for R40W-A.

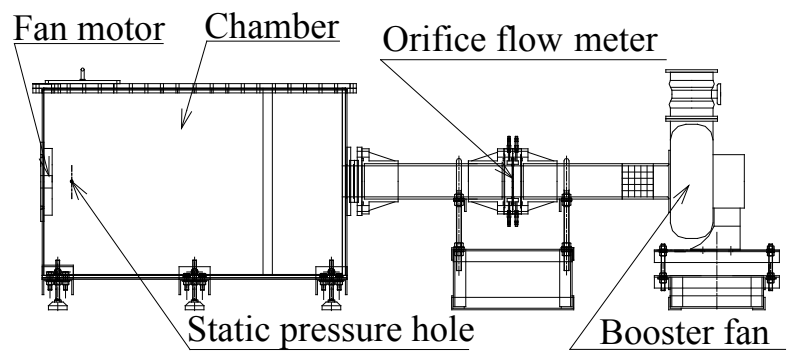

Figure 2. Schematic diagram of experimental apparatus.

Table 1. Primary dimensions of rotors.

\begin{tabular}{ccccc}
\hline & & Hub & Mid & Tip \\
\cline { 3 - 5 } & Diameter $[\mathrm{mm}]$ & 25 & 31.1 & 37.2 \\
\hline \multirow{3}{*}{ Front rotor } & Blade number & & 7 & \\
& Solidity & 1.61 & 1.4 & 1.22 \\
& Stagger angle & $18.2^{\circ}$ & $31.2^{\circ}$ & $36^{\circ}$ \\
\hline \multirow{3}{*}{ Rear rotor } & Diameter $[\mathrm{mm}]$ & 26 & 31.1 & 37.2 \\
& Blade number & & 5 & \\
& Solidity & 1.03 & 1.17 & 1.1 \\
& Stagger angle & $40.6^{\circ}$ & $40^{\circ}$ & $45.5^{\circ}$ \\
\hline
\end{tabular}


experimental apparatus and a picture of the experimental apparatus is shown in Figure 3. The experimental apparatus was designed based on the Japanese Industrial Standard and air blown in a test section passes the rotors, a chamber, a measurement duct and a booster fan and blow out in the ambient atmosphere. Each rotor is driven by a brushless motor set inside of the hub and the motor is supported by spokes connected to the casing. There are three spokes downstream of the front and the rear rotors to support the motors and the casing has curved corners at the inlet and outlet of it and circular diffuser and nozzle between the front and rear rotor as can be confirmed by a sectional view of R40W-A in Figure 4. Therefore, there are some geometrical restrictions for the high pressure and large flow rate small-sized cooling fan. In the performance test, the rotational speed of each rotor was kept constant $\left(N_{f}=15,000 \mathrm{~min}^{-1}, N_{r}\right.$ $=14,000 \mathrm{~min}^{-1}$ ) by PWM control using a function generator and the flow rate controlled by the booster fan and exit valve. The fan static pressure $(\Delta P)$ is measured by a pressure difference between static holes downstream of the rotor installed at the chamber and ambient air. Further, the rotational speed was evaluated using a pulse of the motor measured by an oscilloscope. Flow rates were measured by an orifice meter set at the measurement duct and the pressure curve from a cutoff flow rate to a large flow rate was investigated in the experiment.

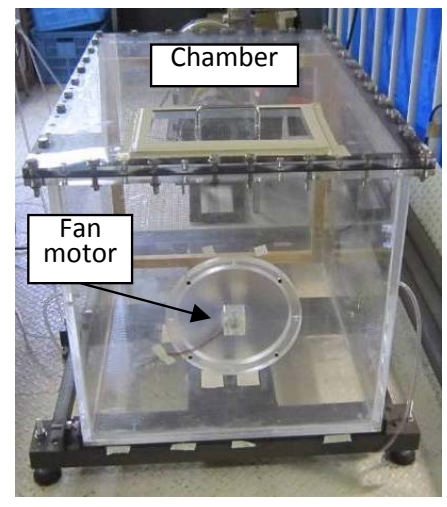

Figure 3. Picture of experimental apparatus.

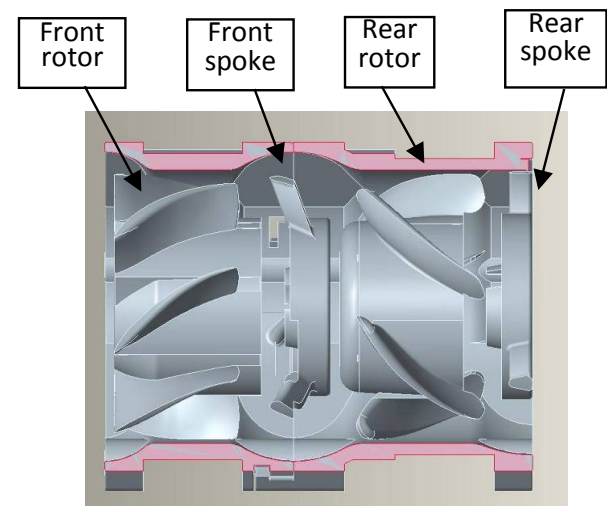

Figure 4. Sectional view of casing for R40W-A. 


\section{Numerical Analysis Conditions}

Commercial software ANSYS-CFX16.2 was used to investigate the flow condition which couldn't be measured by the experiment. Unsteady numerical analysis was conducted using the three dimensional model because the complicated structure existed in this cooling fan. Figure 5 shows the numerical model. In the numerical model, a simplification of the geometry was conducted by extension of the length of the front rotor hub to the rear rotor inlet as shown in Figure 5 in order to reduce the computational cost. Numerical grids used for the numerical analysis are shown in Figure 6. The numerical domains comprise inlet, front rotor, spoke, rear rotor, chamber and outlet duct regions. The numerical grid

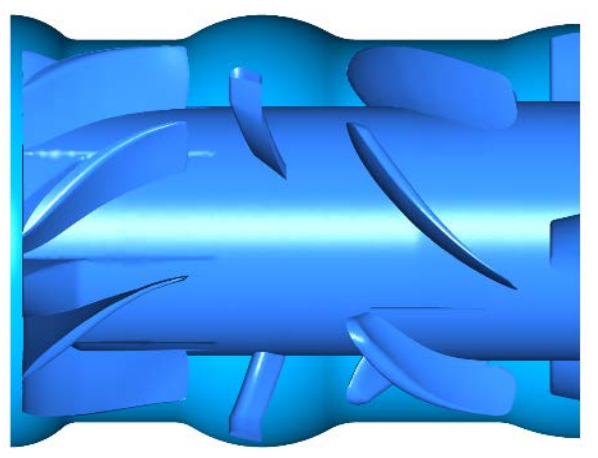

Figure 5. Sectional view of numerical model.

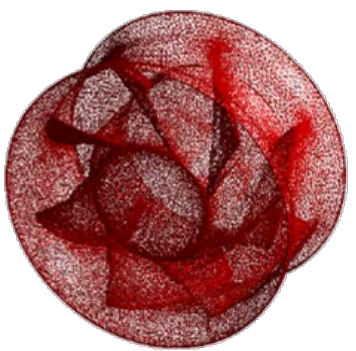

(a)

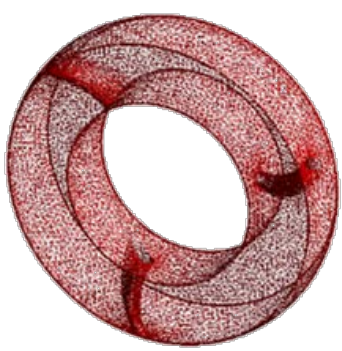

(b)

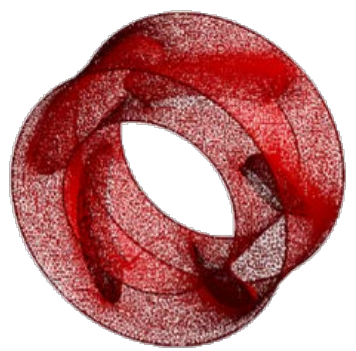

(c)

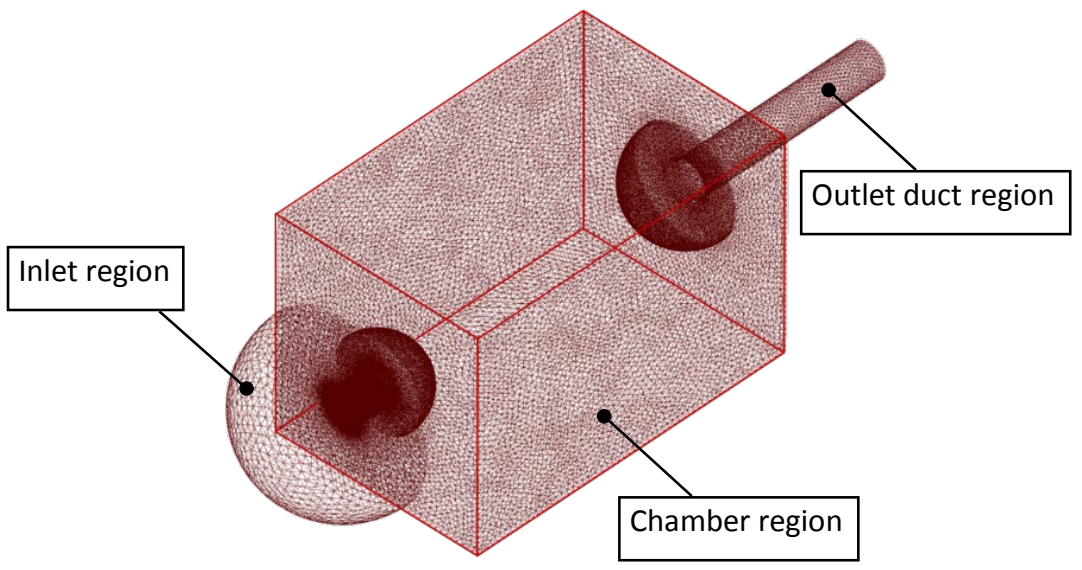

(d)

Figure 6. Numerical analysis grids. (a) Front rotor; (b) Spoke region; (c) Rear rotor; (d) Whole domains. 
points are 617,090 for the inlet region, 1,479,336 for the chamber region and 237,628 for the outlet duct region respectively. The numerical grid points are $3,924,468,362,848$ and 3,107,155 for the front rotor, spoke and rear rotor regions respectively. The tip clearance was kept $0.6 \mathrm{~mm}$ the same as the experimental apparatus in the numerical analysis. At an inlet boundary, constant flow rate was given and constant pressure for an outlet boundary condition. Because the gage pressure $0 \mathrm{~Pa}$ was given at the outlet of the numerical domain (outlet duct region), static pressure and total pressure inside the rotors becomes minus. The coupling between the front and rear rotors was accomplished by the transient rotor stator. The scalable wall function was used as a near wall treatment and the LRR Reynolds Stress model, which provides high accuracy for some complex flows, was used as a turbulence model. The unsteady numerical flow analysis was conducted at the operating flow rate $Q_{o}=0.55 \mathrm{~m}^{3} / \mathrm{min}$ and 4 other flow rate points $0.4 Q_{o}, 0.7 Q_{o}, 1.3 Q_{o}$ and $1.6 Q_{o}$. A time step number per one rotor rotation was 140 and the time step was $t=2.857 \times 10^{-5} \mathrm{~s}$. The data of one rotor rotation were obtained after 10 rotor rotations in the unsteady numerical analysis and the computational time for 10 rotor rotations at each flow rate was about 1 week. The fan static pressure for the numerical analysis was calculated by the static pressure difference between the static pressure hole positions installed at the chamber and the inlet boundary of the numerical domain, which was almost the same with the experiment. The air was assumed incompressive fluid, so the density of the air was constant in this research. The static pressure efficiency was evaluated by a ratio of a multiplication of the static pressure and the flow rate to the shaft power obtained by the numerical analysis.

\section{Experimental and Numerical Analysis Results}

\subsection{Performance Curve of R40W-A}

Figure 7 shows the fan static pressure curves of the test fan at the design rotational speed $N_{f} / N_{r}=15,000 \mathrm{~min}^{-1} / 14,000 \mathrm{~min}^{-1}$ obtained by the experiment. Numerical analysis results are also shown in Figure 7. A horizontal axis is the flow rate $Q$ and a vertical axis is the fan static pressure $\Delta P_{s}$. The fan static pressure of each front and rear rotor obtained by the numerical analysis, in which the static pressure difference in the spoke region is excluded, is also shown in Figure 7. The test fan showed the negative slope of the pressure curve from the operating flow rate $Q_{o}=0.55 \mathrm{~m}^{3} / \mathrm{min}$ to the maximum flow rate $Q=1.08$ $\mathrm{m}^{3} / \mathrm{min}$ and the maximum fan static pressure $\Delta P_{s}=510 \mathrm{~Pa}$ was obtained at the operating flow rate $Q_{o}=0.55 \mathrm{~m}^{3} / \mathrm{min}$ in the experiment. The maximum fan static pressure of the numerical analysis was also obtained at the operating flow rate $Q_{o}=0.55 \mathrm{~m}^{3} / \mathrm{min}$ and its value was $\Delta P_{s}=500 \mathrm{~Pa}$, which was within the $2 \%$ accuracy of the experiment. A positive slope of the pressure curve appeared from the partial flow rate $Q=0.50 \mathrm{~m}^{3} / \mathrm{min}$ to the operating flow rate $Q_{o}=0.55 \mathrm{~m}^{3} / \mathrm{min}$. After that, the pressure curve became flat in partial flow rates region $Q=0.35$ $0.50 \mathrm{~m}^{3} / \mathrm{min}$. Then, the positive slope of the pressure curve appeared again in 


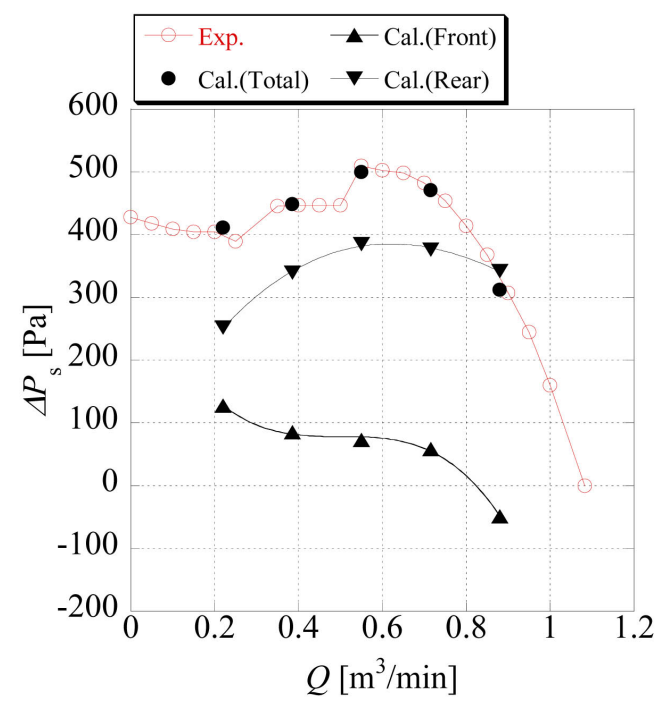

Figure 7. Fan static pressure curves.

partial flow rates region $Q=0.25-0.35 \mathrm{~m}^{3} / \mathrm{min}$. It was confirmed that the operating condition of the test fan was in severe condition as the fan static pressure was set extremely high and the operating flow rate existed near the flow rate range with the positive slope of the fan static pressure curve. The whole of the numerical data accorded with the experimental data and the trend of the fan static pressure curve of the experiment could be captured well by the numerical analysis quantitatively. Then, the performance of each front and rear rotor, which could not be obtained by the experimental method, was investigated by the numerical analysis.

The fan static pressure of the front rotor was extremely lower than that of the rear rotor and the fan static pressure decreased according to the increase of the flow rate. On the other hand, the fan static pressure of the rear rotor was high and about $77 \%$ of the total fan static pressure was obtained by the rear rotor at the operating flow rate $Q_{o}=0.55 \mathrm{~m}^{3} / \mathrm{min}$. The fan static pressure of the rear rotor showed the maximum value at $Q_{o}=0.55 \mathrm{~m}^{3} / \mathrm{min}$, where the maximum total fan static pressure obtained. The static pressure efficiency of each front rotor, rear rotor and its total including static pressure difference in spoke region are shown in Figure 8. The static pressure efficiency was obtained by the unsteady numerical analysis. A horizontal axis is the flow rate $Q$ and a vertical axis is the static pressure efficiency $\eta_{s}$. The maximum total fan static pressure efficiency obtained at the operating flow rate $Q_{o}=0.55 \mathrm{~m}^{3} / \mathrm{min}^{-1}$ was low as $\eta_{s}=46.7 \%$ because of the design specification of high pressure and large flow rate cooling fan with the $40 \mathrm{~mm}$ square casing. The fan static pressure of the rear rotor was higher than that of the front rotor in all flow rates, where numerical analysis conducted, and the difference of the static pressure efficiency between the front and rear rotors became large with the increase of the flow rate. It was found from the performance curve of the test fan that the static pressure increased significantly in the rear rotor and its tendency became large with the increase of the 


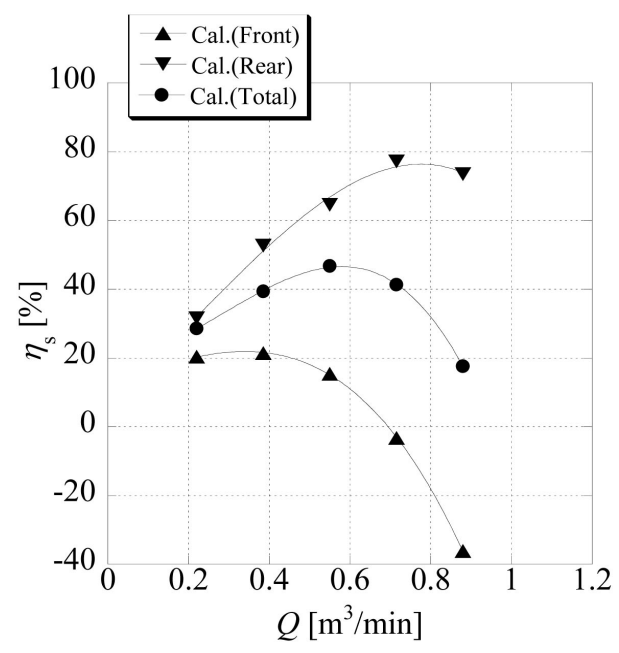

Figure 8. Static pressure efficiency.

flow rate. Therefore, the internal flow condition of each front and rear rotor was investigated using the numerical analysis results to clarify the difference of each front and rear rotor performance.

\subsection{Internal Flow Condition of R40W-A at Operating Flow Rate $Q_{o}$}

The internal flow conditions at the operating flow rate $Q_{o}=0.55 \mathrm{~m}^{3} / \mathrm{min}$ were investigated by the numerical analysis results. Blade-to-blade relative velocity vectors and static pressure distribution at each radial positions $r / r_{c}=0.74, r / r_{c}=$ 0.85 and $r / r_{c}=0.96$ are shown in Figure 9 and Figure 10. The flow rate is the operating flow rate $Q_{o} . r$ and $r_{c}$ means radius, where the data obtained, and inner radius of the casing. It was observed from the internal flow near the hub in Figure 9(a) and Figure 10(a) that the stagnation point at the leading edge of the front and rear rotors existed near the pressure surface of the blade at the operating flow rate $Q_{o}=0.55 \mathrm{~m}^{3} / \mathrm{min}$ and the small separation occurred on the suction surface of the rear rotor. On the other hand, the relative velocity of the suction surface of the rear rotor was high because of the circumferential velocity at the outlet of the front rotor and decreased suddenly at the outlet of the rear rotor. Therefore, the static pressure increased significantly in the rear rotor, although the static pressure increase of the front rotor was small. This flow condition was similar to those at other radial positions in Figure 9(b) and Figure 9(c) and Figure 10(b) and Figure 10(c) and corresponded to the result that the fan static pressure of the rear rotor was large compared to the front rotor in Figure 7. The leakage flow from the pressure surface to the suction surface near the mid of the blade chord were confirmed for both front and rear rotors at shroud region in Figure 9(c). Total pressure distribution at each radial position is shown in Figure 11. The flow rate is the operating flow rate $Q_{o}$. The total pressure decreased downstream of the trailing edge of the front rotor and spoke at $r / r_{c}=0.74$ and significant reduce of the total pressure is observed on the suction surface of the rear rotor due to the small separation on the suction surface of the rear rotor, 


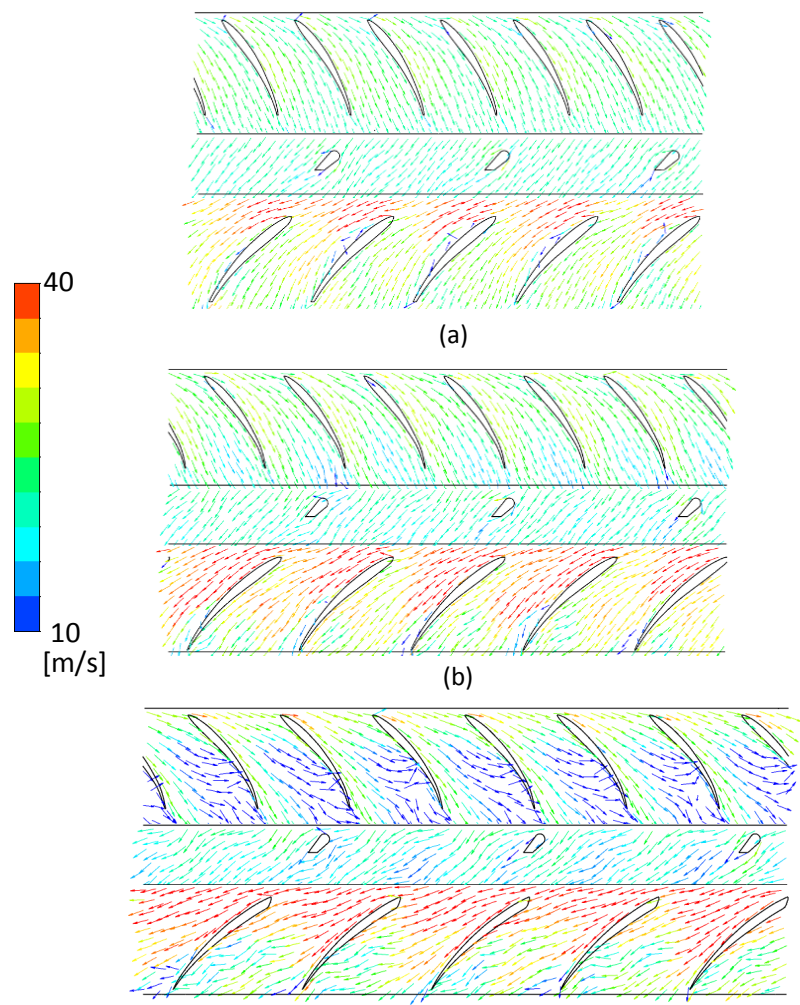

(c)

Figure 9. Velocity vectors at $Q_{o}$. (a) $r / r_{c}=0.74$; (b) $r / r_{c}=0.85$; (c) $r / r_{c}=0.96$.

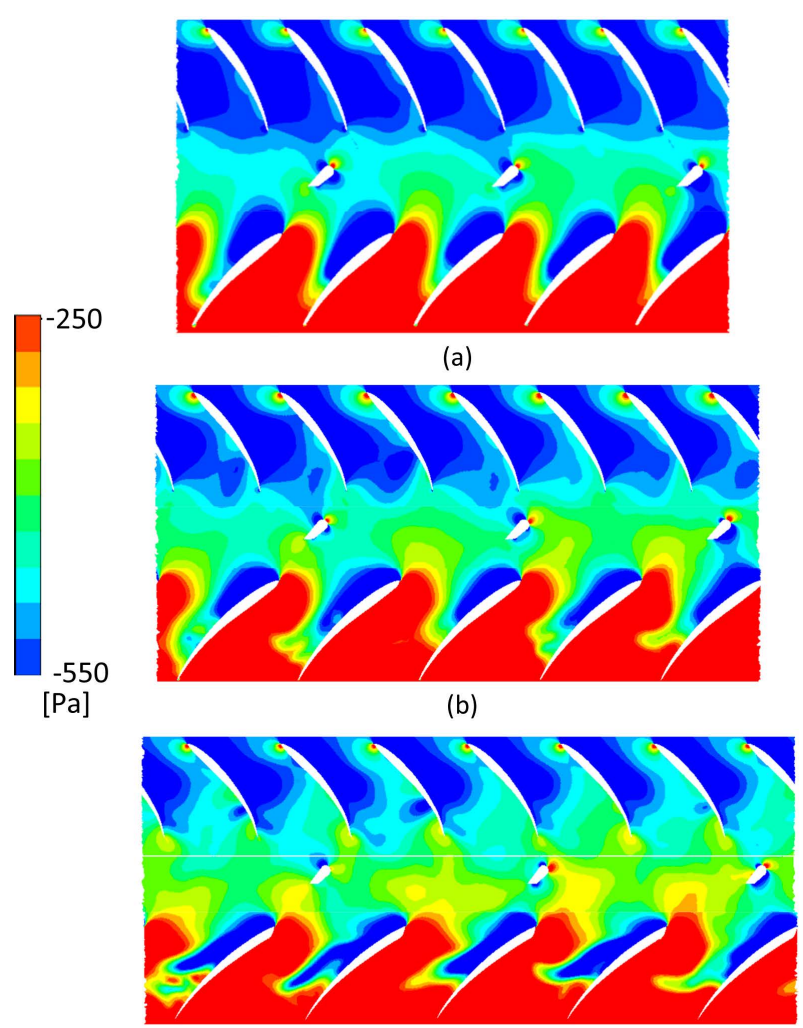

(c)

Figure 10. Static pressure distribution at $Q_{o}$. (a) $r / r_{c}=0.74$; (b) $r / r_{c}=0.85$; (c) $r / r_{c}=0.96$. 


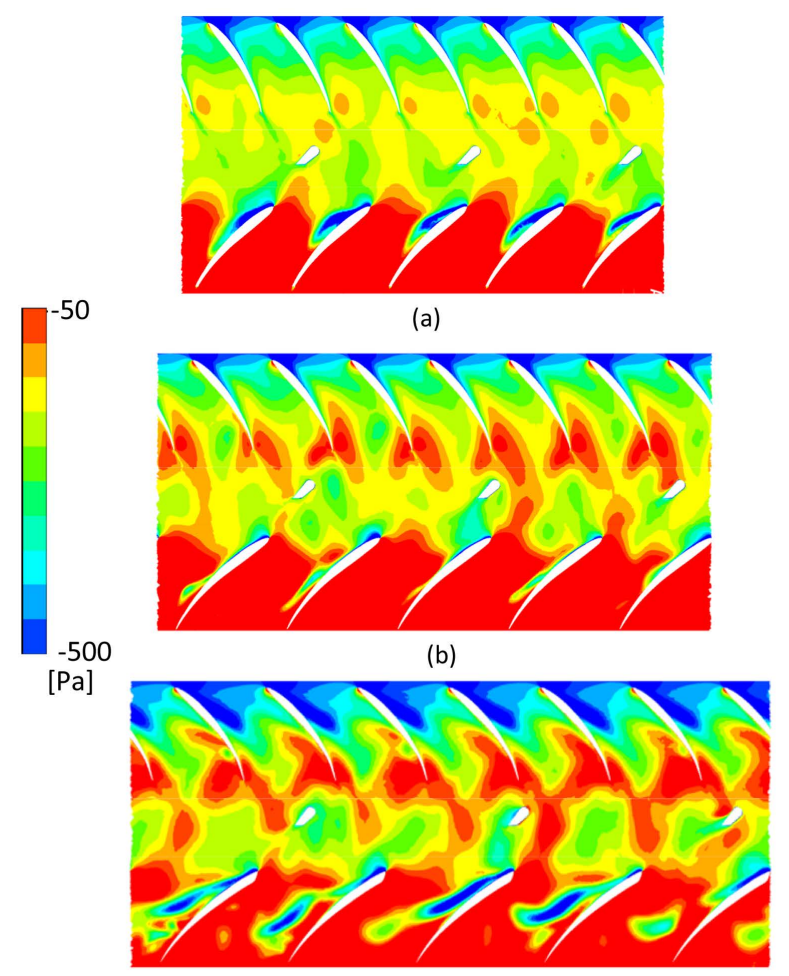

(c)

Figure 11. Total pressure distribution at $Q_{o^{\circ}}$ (a) $r / r_{c}=0.74$; (b) $r / r_{c}=0.85$; (c) $r / r_{c}=0.96$.

which can be confirmed in Figure 9(a). On the other hand, this separation region on the suction surface becomes small and low total pressure region reduces at radial mid position $r / r_{c}=0.85$. The influence of the tip leakage flow is strong for both front and rear rotors near the tip region at $r / r_{c}=0.96$. The low total pressure region associated to the tip leakage flow spreads blade-to-blade region widely for both rotors and the total pressure loss is considered to be large.

The meridional velocity vectors and static pressure on the vertical plane at the operating flow rate $Q_{o}=0.55 \mathrm{~m}^{3} / \mathrm{min}$ are shown in Figure 12 and Figure 13 . The rotational direction of the front rotor is front side of the paper and that of the rear rotor is back side of the paper. The vortex occurred at the inlet corner curve and this vortex could be caused by the separation of the main flow around the inlet corner. In general, the corner separation occurred at the inlet of the casing and this separation vortex influenced on the inlet flow condition. In this test fan, this vortex was basically got stuck in the inlet corner, so the flow condition near the inlet shroud wasn't influenced by this vortex. The leakage flow from the blade tip was observed for both front and rear rotors in Figure 12 and these low velocity regions related to the leakage flow was observed in wide region near the shroud on the meridional plane. There is the circular diffuser and nozzle region on the shroud between the front and rear rotors to improve the performance and noise. The back flow occurred in the diffuser region and the static pressure was high in this region. The low velocity region spread radially inner section between the front and rear rotors by the influence of the back flow 


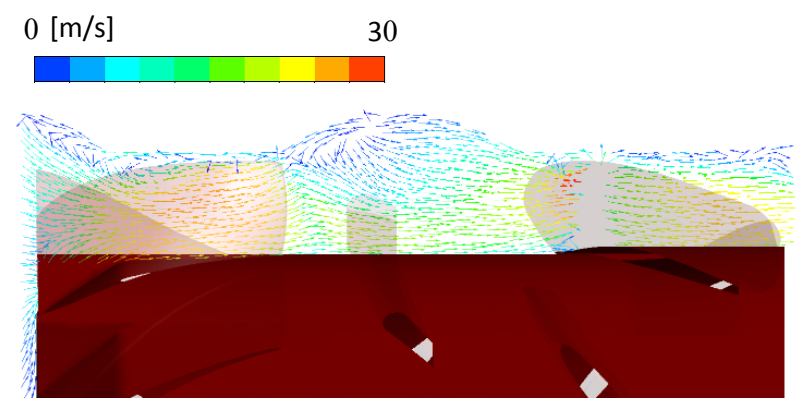

Figure 12. Meridional velocity vectors at $Q_{o}$.

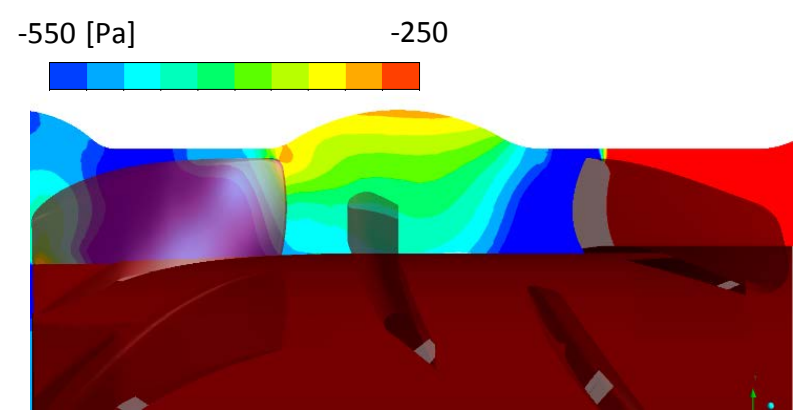

Figure 13. Static pressure distribution at $Q_{O}$.

region. Then, the main flow inclined to radial mid and hub region between the front and rear rotors. The back flow region disappeared in the circular nozzle region with the increase of the velocity and uniform flow was achieved at the inlet of the rear rotor. The static pressure increased significantly in the rear rotor and its distribution was uniform in radial direction as could be seen in Figure 13. Total pressure distribution on the vertical plane at the operating flow rate $Q_{o}$ $=0.55 \mathrm{~m}^{3} / \mathrm{min}$ is shown in Figure 14. The low total pressure region caused by the tip leakage flow is clearly observed near the tip region for both front and rear rotor on the meridional plane and its area is large enough to influence on the main flow of the test cooling fan.

\section{Concluding Remarks}

The performance characteristics of the high pressure and large flow rate small-sized contra-rotating axial flow fan were investigated by the experiment and the numerical analysis. Then, the internal flow conditions at the operating flow rate were clarified by the numerical analysis results. The fan static pressure of the front rotor was extremely lower than that of the rear rotor and the fan static pressure of the front rotor decreased according to the increase of the flow rate. On the other hand, the fan static pressure of the rear rotor was high and about $77 \%$ of the total fan static pressure was obtained by the rear rotor at the operating flow rate $Q_{o}=0.55 \mathrm{~m}^{3} / \mathrm{min}$. The maximum total fan static pressure efficiency obtained at the operating flow rate $Q_{o}=0.55 \mathrm{~m}^{3} / \mathrm{min}$ was low as $\eta_{s}=$ $46.7 \%$ because of the design specification of high pressure and large flow rate cooling fan with the $40 \mathrm{~mm}$ square casing. The fan static pressure of the rear rotor 


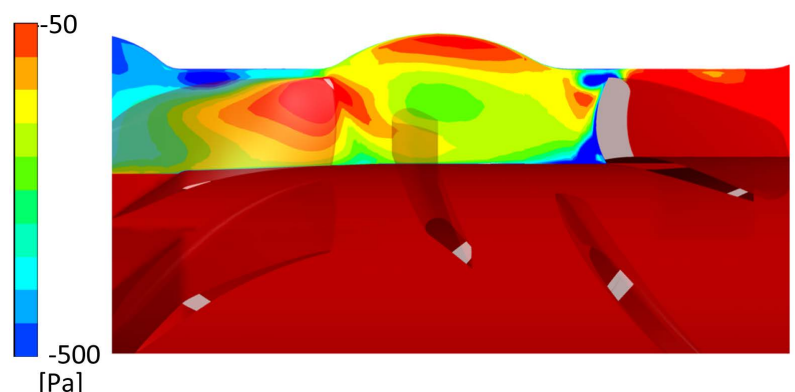

Figure 14. Total pressure distribution at $Q_{o}$.

was higher than that of the front rotor in all flow rates. The stagnation point at the leading edge of the front and rear rotors existed near the pressure surface of the blade at the operating flow rate $Q_{o}=0.55 \mathrm{~m}^{3} / \mathrm{min}$. On the other hand, the relative velocity of the suction surface of the rear rotor was high because of the circumferential velocity at the outlet of the front rotor and decreased suddenly at the outlet of the rear rotor. Therefore, the static pressure increased significantly in the rear rotor, although the static pressure increase of the front rotor was small. In general, the corner separation occurred at the inlet of the casing and this separation vortex influenced on the inlet flow condition. In this test fan, this vortex was basically got stuck in the inlet corner, so the flow condition near the inlet shroud wasn't influenced by this vortex. There is the circular diffuser and nozzle region on the shroud between the front and rear rotors. The back flow occurred in the diffuser region and the static pressure was high in this region. The low velocity region spread radially inner section between the front and rear rotors by the influence of the back flow region. Then, the main flow inclined to radial mid and hub region between the front and rear rotors. The back flow region disappeared in the circular nozzle region with the increase of the velocity and uniform flow was achieved at the inlet of the rear rotor. The static pressure increased significantly in the rear rotor and its distribution was uniform in radial direction. The low total pressure region caused by the tip leakage flow was clearly observed near the tip region for both front and rear rotor on the meridional plane and its area was large enough to influence on the main flow of the test cooling fan.

\section{Acknowledgements}

The authors wish to show our special thanks to the supports by Nidec Corporation, the project research aid from Tokushima University, Japan Science and Technology Agency and Komiya research aid.

\section{References}

[1] Hata, T. and Nakamoto, S. (2010) Energy Saving Service for Data Centers. Journal the Japan Society for Precision Engineering, 76, 272-275. https://doi.org/10.2493/jjspe.76.272

[2] Miyahara, M. and Fukano, T. (2006) Fan Cooling Technology for Small Electronic 
Device. Turbomachinery (in Japanese), 34, 129-134.

[3] Ito, T., Minorikawa, G. and Fan, Q. (2009) Experimental Research for Performance and Noise of Small Axial Fan. International Journal of Fluid Machinery and Systems, 2, 136-146. https://doi.org/10.5293/IJFMS.2009.2.2.136

[4] Liu, P., Shiomi, N., Kinoue, Y., Setoguchi, T and Jin, Y. (2014) Effect of Inlet Geometry on Fan Performance and Inlet Flow Fields in a Semi-Opened Axial Fan. International Journal of Fluid Machinery and Systems, 7, 60-67.

https://doi.org/10.5293/IJFMS.2014.7.2.060

[5] Furukawa, A., Shigemitsu, T. and Watanabe, S. (2007) Performance Test and Flow Measurement of Contra-Rotating Axial Flow Pump. Journal of Thermal Science, 16, 7-13. https://doi.org/10.1007/s11630-007-0007-4

[6] Furukawa, A., Cao, Y., Okuma, K. and Watanabe, S. (2000) Experimental Study of Pump Characteristics of Contra-Rotating Axial Flow Pump. Proceeding 2nd International Symposium on Fluid Machinery and Fluid Engineering, 67, 245-252.

[7] Kodama, Y., Hayashi, H., Fukano, T and Tanaka, K. (1994) Experimental Study on the Characteristics of Fluid Dynamics and noise of a Counter-Rotating Fan (1st Report, Effects of the Supporter Shape of the Electric Motor and the Distance between Two Rotors on the Characteristics. Transactions of the JSME (in Japanese), 60, 2764-2771.

[8] Nouri, H., Ravelet, F., Bakir, F., Sarraf, C. and Rey, R. (2012) Design and Experimental Validation of a Ducted Counter-Rotating Axial-Flow Fans System. Journal of Fluids Engineering, 134, 104504. https://doi.org/10.1115/1.4007591

[9] Shigemitsu, T., Fukutomi, J. and Agawa, T. (2013) Performance Characteristics and Flow Conditions of High Pressure and Large Flow Rate Cooling Fan. Proceedings of the ASME Fluids Engineering Division Summer Meeting, Lake Tahoe, 7-11 July 2013, FEDSM2013-16273. https://doi.org/10.1115/FEDSM2013-16273

[10] Shigemitsu, T., Fukutomi, J. and Agawa, T. (2013) Internal Flow Condition of High Power Contra-Rotating Small-Sized Axial Fan. International Journal of Fluid Machinery and Systems, 6, 25-32. https://doi.org/10.5293/IJFMS.2013.6.1.025

[11] Shigemitsu, T., Furukawa, A., Okuma, K. and Watanabe, S. (2002) Experimental Study on Rear Rotor Design in Contra-Rotating Axial Flow Pump. Proceedings of 5th JSME/ KSME Fluids Engineering Conference, Nagoya, 17-21 November 2002, 1453-1548.

[12] Ravelet, F., Bakir, F., Sarraf, C. and Wang, J. (2018) Experimental Investigation on the Effect of Load Distribution on the Performances of a Counter-Rotating Axial-Flow Fan. Experimental Thermal and Fluid Science, 96, 101-110. https://doi.org/10.1016/j.expthermflusci.2018.03.004

[13] Wang, C. and Huang, L. (2014) Passive Noise Reduction for a Contrarotating Fan. Journal of Turbomachinery, 137, 031007. https://doi.org/10.1115/1.4028357

[14] Sanders, A.J., Papalia, J. and Fleeter, S. (2002) Multi-Blade Row Interactions in a Transonic Axial Compressor: Part I - Stator Particle Image Velocimetry (PIV) Investigation. Journal of Turbomachinery, 124, 10-18. https://doi.org/10.1115/1.1411973

[15] Nouri, H., Danlos, A., Ravelet, F., Bakir, F. and Sarraf, C. (2013) Experimental Study of the Instationary Flow between Two Ducted Counter-Rotating Rotors. Journal of Engineering for Gas Turbines and Power, 135, 022601. https://doi.org/10.1115/1.4007756

[16] Shigemitsu, T., Fukutomi, J. and Okabe, Y. (2010) Performance and Flow Condition 
of Small-Sized Axial Fan and Adoption of Contra-Rotating Rotors. Journal of Thermal Science, 19, 1-6. https://doi.org/10.1007/s11630-010-0001-0 